\title{
ON THE EFFICIENCY OF THE SOURCE OF ELECTROMAGNETIC EMISSION IN THE ELECTRON DIFFUSION REGION FORMED BY PLASMA FLOW
}

\author{
V. M. Gubchenko*
}

\begin{abstract}
Extended Abstract
There is a certain similarity between inductive mechanisms working with the power $P$ at low sub-critical frequencies $\omega \sim 1 / t<<\omega_{p}$ ( $\omega_{p}$ as plasma frequency) forming asymmetric magnetized astrophysical large scales $r \sim 1 / k$ ( $k$ as wave number) magnetic structures (e.g., planetary magnetosphere's magnetotails, heliospheric current sheets, solar/stellar corona streamers/jets, etc.) and those magnetic structures in high energy dense (HED) laboratory laser plasmas. Both are formed by hot collisionless plasma flows with the velocity $v^{\prime}$, moving relative to the magnetization $\mu(\vec{X})$ distributed on the scale $r_{0}$, which is considered here as a resting quasi-particle.

These 3D structures are formed by electromagnetic energy generation by a hot collisionless plasma flow providing the power $P$, and this power is directed to the acceleration of energetic electrons, which further transmit the power at overcritical high frequencies $\left(\omega_{\text {rad }}>>\omega_{p}>>\omega\right)$ and small scales $\lambda<<r$ as electromagnetic (e.m.) emissions process with the power $P_{\text {rad }}<P$. The particles are receiving energy with the power $P$ from the continuous dissipative collisionless magnetic reconnection process, which is located in the $3 \mathrm{D}$ electron asymmetric diffusion region (DR) with its magnetic structure self-consistent with this dissipation as acceleration. In the natural conditions $P_{\mathrm{rad}} / P<1$ and in the conditions of the laser plasma experiments we are directed to get conditions $P_{r a d} \rightarrow P$. In both of these cases we need to estimate $P$ as the upper limit for the radiation power $P_{r a d}$ formed in the plasma flow and find a way to increase $P$ via the impedance $Z$ as the "efficiency" parameter.

These electromagnetic processes of plasma expansion with the powers $P$ and $P_{\text {rad }}$ are separated by the frequencies $\omega<<\omega_{\text {rad }}$ and the scales $1 / k>>\lambda$ and are studied separately by different models in plasma physics. Our task is to make a step towards their unification and to estimate the power $P$, which is provided from the DR by the magnetic reconnection process to the energetic particles selfconsistently, and to get the electromotive force (e.m.f.) $E_{e m f}$ which drags the plasma flow and decreases the energy $U$ of the escaping ions with charge $q$.

The stationary magnetic structures formed by direct plasma flow are observed as transition from the initial radially fastly falling, "nonradiating" asymptotics to the
\end{abstract}

*Institute of Applied Physics of the Russian Academy of Sciences, Nizhny Novgorod, Russia 
much slower "emitting" type asymptotics in the "wave zones". The "wave zones" of the plasma e.m. mode emission (inductive fields, Alfvén waves, and e.m. waves) appear out of the magnetization characterizing the electromagnetic dissipation by different "radiation" modes. These "wave zones" appear non-stationary with oscillations $\omega \neq 0$, or stationary with $\omega=\vec{k} \overrightarrow{v^{\prime}} \neq 0$ - the observer is in the reference system of the stationary flowing plasma. The transition from near magnetization fields to the different modes "wave zones" asymptotics forms the 3D DR of magnetic reconnection by magnetic tails and e.m. radiation cones. The zone here created by the inductive field plasma mode, where dissipation with the power $P$ is due to the free acceleration of electrons and ions by the inductive field, is called the electron DR. The asymmetry of the DR is provided by two factors: plasma direct flow and asymmetry in the magnetization $\vec{\mu}$.

The 3D asymmetric electron diffusion (dissipative) magnetic reconnection region (DR) contains electrons and ions, weakly magnetized with $r<<r_{c e}\left(r_{c e}\right.$ is the electron cyclotron radius) by normal to the flow magnetic field $\overrightarrow{B_{n}}$. In the hot plasma one group of particles ("nonresonant" ones) in the velocity phase space is reflected by the magnetic field $\vec{B}=\nabla \times \vec{A}$ providing diamagnetism $\mu<1$; and the other group of particles ("resonant" ones) are free to be accelerated by the electric field $\vec{E}=-(1 / c) \partial \vec{A} / \partial t$ which provides ohmic dissipation for magnetic reconnection.

The bulk flow velocity $v^{\prime}$ is prescribed externally in our approach. In plasma kinetics the flow is originated by ambipolar subsonic and supersonic hot plasma expansion, which is selfconsistent with the potential field $\varphi$ providing electrostatic drag on plasma electrons and acceleration on plasma ions with the charges $q=e, i$; this electrostatic stationary process is considered as plasma polarization without dissipation. The plasma bulk is in the hot electron regime $v^{\prime}<<v_{e}$, where $v_{e}$ is the electron thermal velocity. This ambipolar potential $\varphi$ action is modified by the inductive electromotive force (electrotechnical e.m.f.) $E_{\text {emf }}$, provided by ampere forces work defined via the vector potential $\vec{A}$, and we get the effective potential $\varphi_{e f f}=\varphi-E_{e m f}$. This provides a decrease of the energy $\Delta U=q E_{e m f}$ of the escaping ions with charge $q>0$.

The magnetization $\vec{\mu}(\vec{X})=\mu_{d}(\vec{X})+\mu_{\tau}(\vec{X})$ is formed by superposition of the dipole $\mu_{d}(\vec{X})$ and the toroid $\mu_{\tau}(\vec{X})$ components, forming an asymmetric "coil" with the current density $\overrightarrow{j_{0}}=c \nabla \times \vec{\mu}(\vec{X})$ and the integral current $I$ distributed on scale $r_{0}$, which provides a configuration with the null point of the magnetic field in vacuum and the normal component $B_{n}$. The dipole and toroid are orthogonal to each other and to the plasma flow velocity, $|\mu(\vec{X})| \sim \exp \left(-r^{2} / 2 r_{0}^{2}\right)$.

The dissipation power $P=\overrightarrow{F_{A}} \overrightarrow{v^{\prime}}=\int d^{3} x\left(\overrightarrow{j_{0}} \vec{E}\right)=E_{e m f} I$ going to the particle's acceleration by inductive fields is defined as the work of the Ampere forces $\operatorname{drag} \overrightarrow{F_{A}}$ over the flow, which is equal to work of the eddy field $\vec{E}$ on the eddy currents $\overrightarrow{j_{0}}$.

Note that we used an analytical approach to the DR modeling as a kinetic 3D problem, which operates with the Vlasov kinetic equation with different behaviors of the group of the "resonant" and the group of the "nonresonant" electrons in the plasma flow. The alternative analytical approaches to the physics of the asymmetric electron DR were reviewed recently to apply it to the MMS mission: They are limited now by electron MHD with electron pressure tensor, ignoring the "resonance" particles dissipative kinetic key effect originated by the direct plasma flow.

For the "wave zone" formation by a flow with the velocity $\overrightarrow{v^{\prime}}$ we have an e.m. dissipation with the power $P$ via direct e.m. radiation or absorption in plasma (par- 
ticle acceleration) in a wide range of frequencies $\omega=\vec{k} \overrightarrow{v^{\prime}}$ and scales $r \approx 1 / k$, when $\overrightarrow{v^{\prime}}>v_{p h}=\omega_{r} / k$ here $v_{p h}=\omega_{r} / k$ - the phase speed the eigen-modes in the plasma takes place. For for the modes of the inductive electromagnetic field $v_{p h}=\omega_{r} / k=0$. This means that the inductive type of the "wave zone" - the dissipative tail/wake - develops at any velocity $v^{\prime} \neq 0$ and the electron DR forms near the source. The mode decrement $\omega_{i}(k)$ provides collisionless absorption with the power $P$, which goes to the acceleration of plasma particles. Therefore, the magnetosphere-like structure as the electron DR forms, above all, first in an inductive way. Only then, as the velocity is higher, there is the possibility to form the radiative part of the DR structure where the power $P$ goes also to the radiating modes: Alfvén $\left(v^{\prime}>v_{A}\right)$ and e.m. $\left(v^{\prime}>c / n\right.$, where $n$ is the refractive index) waves with high phase velocities $v_{p h}=\omega_{r} /|\vec{k}| \neq 0$.

Collisionless electrons and ions in the inductively formed DR by the flow $v^{\prime}$ are weakly magnetized $\left(r<<r_{c e}\right)$ : Plasma in the DR is unmagnetized and can be presented by the well-known solution of the Vlasov equation for isotropic plasma in terms of the tensor of dielectric permittivity $\epsilon_{i j}(\omega, \vec{k})$. The tensor has only diagonal components: $\epsilon_{l}(\omega, \vec{k})$, responsible for the ambipolar $\varphi$ fields, and $\epsilon_{t}(\omega, \vec{k})$, responsible for the eddy e.m. $\vec{A}$ fields. The plasma e.m. modes described by the dispersion curves $\omega(k)$ are solutions of the equation $D_{T}(\vec{k}, \omega)=1-\omega^{2} \epsilon_{t}(\omega, \vec{k}) /(c k)^{2}=0$, where $\epsilon_{t}(\omega, \vec{k})=\operatorname{Re}\left[\epsilon_{t}(\omega, \vec{k})\right]+i \operatorname{Im}\left[\epsilon_{t}(\omega, \vec{k})\right]$. Here $\operatorname{Re}\left[\epsilon_{t}(\omega, \vec{k})\right] \neq 0$ is related with dissipation-less polarization currents of the "non-resonant" particles, and $\operatorname{Im}\left[\epsilon_{t}(\omega, \vec{k})\right]$ $\neq 0$ is a kinetic effect which is related with currents of the "resonantly" accelerated particles, providing here collisionless dissipation with the power $P$.

The solution of $D_{T}=0$ as $\omega(k)=\omega_{r}+i \omega_{i}$, well-known for the MHD collisionless plasma limit $\omega / k v_{\alpha}>>1$, is a fast e.m. wave $\left(\omega_{r}(k) \neq 0, \omega_{i}=0\right)$ when the thermal velocity of the plasma components $v_{\alpha} \rightarrow 0$. The solution is represented often by the squared refractive index $n^{2}(\omega)=\left(c k / \omega_{r}\right)^{2}$ function. Here $\operatorname{Im}\left[\epsilon_{t}(\omega, \vec{k})\right]=0$, and the dissipation power to the acceleration is $P=0$.

The slow dissipative e.m. process (the inductive mode solution $D_{T}=0$ ) of the DR formation is located in the $n^{2}(\omega)=(c k / \omega)^{2} \rightarrow-\infty$ resonance, inside a narrow resonance line near the frequency $\omega_{0}=0$. Inside the line $\omega / k v_{\alpha}=v^{\prime} / v_{\alpha}<<1$, it is the hot plasma flow kinetic regime under study here. The electron DR requires kinetic consideration, where $\operatorname{Im}\left[\epsilon_{t}(\omega, \vec{k})\right] \neq 0, \omega_{r}=0$ and $\omega_{i}(k) \neq 0$, the resonance absorption appears and we get $P \neq 0$.

The slow process is responsible for the inductive formation of the 3D DR e.m. structure, which is selfconsistent with the generation of accelerated particles mainly electrons. It appears as a kind of a "dark", invisible, absorption process when $n^{2}(\omega)<0$ (a so-called subcritical e.m. regime).

The magnetic field of the $3 \mathrm{D}$ DR region $\vec{B}\left(\vec{x}, \overrightarrow{v^{\prime}}, t\right)$, generated by a direct flow $\overrightarrow{v^{\prime}}$, can be represented as a package of the traveling waves $\exp (i \vec{k} \vec{x}-i \omega t)$ with $\omega=\vec{k} \overrightarrow{v^{\prime}}$. We get the Fourier amplitudes: $\vec{A}_{\vec{k}}, \vec{B}_{\vec{k}}=i\left[\vec{k} \times \vec{A}_{\vec{k}}\right]$ and $\vec{j}_{\vec{k}}=i\left[\vec{k} \times \vec{B}_{\vec{k}}\right] c /(4 \pi)$.

After solving of the Maxwell equation with the magnetization $\vec{\mu}$ as a moving quasi-particle in plasma we get $\vec{A}_{\vec{k}}=\vec{A}_{\mu, \vec{k}}+\vec{A}_{\tau, \vec{k}}$ where $\vec{A}_{\mu, \vec{k}}(\vec{k})=\mu_{0} i\left(k_{y} \overrightarrow{x_{0}}-\right.$ $\left.k_{x} \overrightarrow{y_{0}}\right) M_{G \vec{k}}$ and $\overrightarrow{A_{\tau, \vec{k}}}=-\tau_{0}\left(\vec{k} k_{y}-k^{2} \overrightarrow{y_{0}}\right) M_{G \vec{k}}$.

Here $M_{G \vec{k}}\left(\vec{k}, R e_{m}, G_{V}\right)=4 \pi /(2 \pi)^{3} \exp \left(-1 / 2 k^{2} r_{0}^{2}\right) / k^{2} D_{T}\left(\vec{k}, \vec{k} \overrightarrow{v^{\prime}}\right)$ is a characteristic Green function for the $\vec{\mu}(\vec{X})$ with dipole $\mu_{0} \vec{z}_{0}$ and toroid $\tau_{0} \vec{y}_{0}$ moments. For the regime $\omega / k v_{\alpha}=v^{\prime} / v_{\alpha}<<1$ we get $D_{T}\left(\vec{k}, \vec{k} \vec{v}^{\prime}\right)=1-v^{\prime 2} / c^{2}+k^{-2} r_{D M}^{-2}-i k^{-2} r_{G}^{-2}$. Here $r_{G}, r_{D M}$ are the spatial plasma e.m. scales induced by the bulk plasma flow 
with the velocity $v^{\prime}$. Calculations of the scales $r_{G}, r_{D M}$ for plasma flows with asymmetry and anisotropy of the particle velocity distribution function (PDF) are on http://www.vniitf.ru/images/zst/2012/s3/3-13.pdf.

The elliptical polarization of the fields $\vec{A}_{\vec{k}}, \vec{B}_{\vec{k}}$, and $\vec{j}_{\vec{k}}$ in the 3D DR is related to the different orders of the spatial derivatives defining the currents from the dipole $\overrightarrow{\mu_{d}}$ and the toroid $\overrightarrow{\mu_{\tau}}=\nabla \times \vec{\tau}$ components of the magnetizations $\vec{\mu}(X)$.

For certain directions of the vector $\vec{k}$ we obtain in the 3D DR a circular polarized magnetic field forming a "forceless" Fourier separatrix $f_{\vec{k}}=1 / c\left[\vec{j}_{\vec{k}} \times \vec{B}_{\vec{k}}^{*}\right]_{=} 0$ of the positive and negative force spectral components in the space $\vec{k}$. For another certain direction of the vector $\vec{k}$ we get linearly polarized spectral components - "forced" components in the 3D DR.

We get a similarity of the e.m. dimensionless plasma parameters of the hot flowing collisionless space and laser plasma characterized by the anisotropy and asymmetry of the PDF (linear $G_{V}=r_{G}^{2} / r_{D M}^{2}-$ e.m. quality of plasma flow, nonlinear $\Gamma_{B}=G_{V} / M_{A}^{2}-$ e.m. magnetic "friction" number and $M_{A}=v^{\prime} / v_{A}-$ Alfvénic Mach number), whereas the absolute values of plasma parameters in the considered phenomena might be rather different.

The power spent for the formation of the DR is self-consistent with electron acceleration and determined by the formula $P=E_{e m f} I=I^{2} \operatorname{Re}[Z]$, where $R_{\sum}=\operatorname{Re}[Z]$ as "efficiency" is the active part of the DR impedance $Z$. The power $P$ is "normalized" to the coil current $I=I_{\mu} \sqrt{1+\Gamma_{\tau \mu}^{2}}$, which is defined via the magnetization moments $\mu_{0}=2 \pi r_{0}^{2} I_{\mu} / c$ and $\tau_{0} \approx(4 / 3) \pi r_{0}^{3} I_{\tau} / c$, where $\Gamma_{\tau \mu}=I_{\tau} / I_{\mu}$ is a measure of the toroidality in the external magnetization, $I_{\mu}$ and $I_{\tau}$ - dipole and toroidal currents.

We get the power $P\left(G_{V}, R e_{m}\right)=\int d^{3} x\left(\overrightarrow{j_{0}} \vec{E}\right)=P_{\mu}+P_{\tau}-2 P_{\tau \mu}=I_{\mu}^{2}\left(R_{\mu}+\right.$ $\left.\Gamma_{\tau \mu}^{2} R_{\tau}-2 \Gamma_{\tau \mu} R_{\mu \tau}\right)$, where the power $P_{\mu}$ is related with the ampere forces action on the isolated magnetic dipole. The power $P_{\tau}$ is the action on the isolated magnetic toroid, and $P_{\tau \mu}$ is the crossed action between dipole and toroid components due to the presence of a force-free component in the 3D DR. In particular, for $G_{V} \rightarrow 0$ and $\Gamma_{\tau \mu} \rightarrow 0$ we get $R_{\sum}=R_{\mu}=\frac{P_{\mu}}{I_{\mu}^{2}}=\frac{v^{\prime}}{c^{2}} \frac{2}{(2 \pi)^{2}}\left\{\frac{4}{3 \epsilon}-\frac{12}{\epsilon^{3}}+\frac{5}{\epsilon}-\frac{3 \pi}{2 \epsilon^{2}}-\frac{48}{\epsilon^{3}}+\frac{30 \pi}{\epsilon^{4}}+\right.$ $\left.[c i(\epsilon) \sin (\epsilon)-s i(\epsilon) \cos (\epsilon)]\left(-4+\frac{33}{\epsilon^{2}}-\frac{60}{\epsilon^{4}}\right)+[c i(\epsilon) \sin (\epsilon)+s i(\epsilon) \cos (\epsilon)]\left(\epsilon-\frac{13}{\epsilon}+\frac{60}{\epsilon^{3}}\right)\right\}$, where $R e_{m}=r_{0} / r_{G}=\epsilon^{(1 / 2)}$ is the kinetic collisionless magnetic Reynolds number.

We calculate the "electro-motive force" $E_{\text {emf }}=I R e[Z]=I_{\mu}\left(R_{\mu}+\Gamma_{\tau \mu}^{2} R_{\tau}+\right.$ $\left.2 \Gamma_{\tau \mu} R_{\mu \tau}\right)$ as part in the the effective potential $\varphi_{\text {eff }}=\varphi-E_{\text {emf }}$ providing the decrease $\Delta U=q E_{\text {emf }}$ of the energy $U=q \varphi_{\text {eff }}$ of the escaping charge $q$.

Acknowledgments. This work was supported in part by the Russian Foundation for Basic Research (project Nos. 16-02-00373 and 17-02-00091), and by the Ministry of Education and Science of the Russian Federation (contract No. 14.Z50.31.0007). V.M. G. thanks the organizers of PRE 8 for a wonderful meeting, and the Province of Styria, the Austrian Ministry for Transport, Innovation and Technology, and the Space Research Institute of the Austrian Academy of Sciences for the financial support of his visit to Austria. V.M. G. has written this article in memory of his father M. M. G. 